\title{
When it takes two to tango, FDA suggests a new regulatory dance
}

Two drugs taken together can sometimes be greater than the sum of their parts. And for certain diseases, including cancer, drug combinations are essential to achieve any therapeutic benefit. But the existing regulatory framework for testing and approving new drugs focuses primarily on the effectiveness and safety of individual experimental compounds. This means that it's been nearly impossible to conduct early clinical trials on multiple new drugs at the same time.

All that might soon change. In December, the US Food and Drug Administration (FDA) released draft guidance outlining a path toward developing combinations of unapproved drugs. "We need to enable these innovative targeted therapies in a way that isn't possible in traditional drug development," Janet Woodcock, director of the FDA's Center for Drug Evaluation and Research (CDER), told Nature Medicine.

"The places we think are most likely to have interest, at least initially, are oncology and infectious diseases," says CDER deputy director for clinical science Robert Temple. That's because pathogens such as tuberculosis and HIV quickly develop drug resistance when only single-agent therapies are used. Similarly, tumors often harbor multiple gene mutations that allow cancer to grow and that need to be targeted simultaneously.

Combination drug development at present usually involves phase 3 trials in which fixed doses of two drugs are tested both alone and in tandem against a placebo treatment. To get to that stage, however, experimental drugs usually need to be taken through earlier-stage trials in isolation, even if they have limited utility on their own. The new proposed guidelines would allow drug makers to forego monotherapy trials and conduct combined drug studies earlier in the development process, when there are compelling reasons and preclinical data to support such a move.

Jeff Allen, executive director of Friends of Cancer Research, a Washington, DCbased think tank that cohosted a meeting on combination drug development in September 2009 , says that advances in genomics and personalized medicine have precipitated the need for a new drug development model for these combinations. "It's really the direction the biology is moving," he says. "It becomes much more biologically plausible that two or more agents used in combination could have more efficacy than one agent used alone."

"As our understanding of the molecular biology of cancer continues to increase, we're

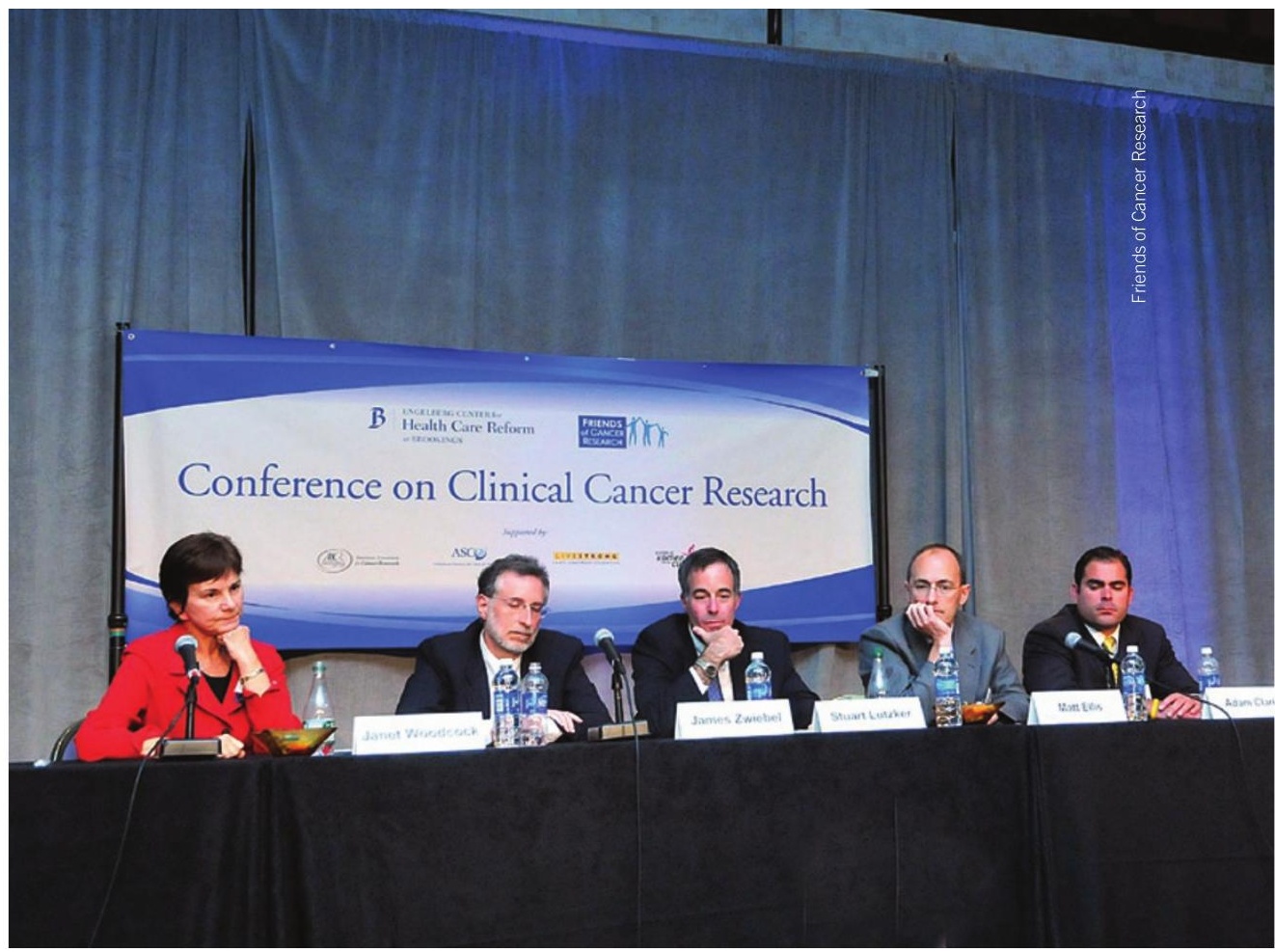

Combining forces: Experts meet in September 2009 to discuss combination drug trials.

seeing that cancers are really being subdivided for different pathways," says Adam Clark, director of scientific and federal affairs at FasterCures, a Washington, DC-based think tank, and lead author on a study published last year outlining the need for a new regulatory approval process (Oncologist 15, 496-499, 2010). "Being able to develop combination therapies that can target two pathways at once or two players in the same pathway will open up more lines of effective therapies for patients based on their genotypes."

\section{Compelling rationale}

The concurrent testing of new drugs does present added risks to study participants, so the FDA is not taking the approach lightly. However, by asking for a nonspecific "compelling biological rationale" from companies wishing to go down the codevelopment pathway, the agency is keeping the wording intentionally vague. "We're trying definitely not to be rigid about this," says Temple. "We're talking about drugs that in the end are supposed to make a difference to a very bad disease. That tends to make one a little bit flexible."

The drug industry, for the most part, is embracing the FDA's new guidance. "You'd like to push the novel combination strategy earlier in the life cycle of the drug development process," says Stuart Lutzker, vice president of BioOncology Exploratory Clinical Development at Genentech in South San Francisco and a coauthor of the Oncologist report. But even without the policy being fully formalized, last year Genentech started recruiting participants with advanced solid tumors for an early-stage trial involving two drugs, one that selectively inhibits MEK, also known as mitogen-activated protein kinase, and another that blocks the product of an oncogene called phosphoinositide 3-kinase (PI3-kinase). Trials are also planned to test the former drug in combination with a BRAF kinase inhibitor for metastatic melanoma and to try the latter drug in conjunction with an investigational targeted agent to treat breast cancer.

Similarly, GlaxoSmithKline is testing its own MEK and PI3-kinase double whammy treatment as well as running trials combining the same MEK inhibitor with another drug that blocks part of the PI3-kinase pathway. "And the fact that the FDA has come out with the draft guidance document is going to encourage more companies to do the same," Lutzker says.

Elie Dolgin 